\title{
Evolution and Function of Thioester- Containing Proteins and the Complement System in the Innate Immune Response
}

\author{
Upasana Shokal and loannis Eleftherianos* \\ Department of Biological Sciences, The George Washington University, Washington, DC, United States
}

The innate immune response is evolutionary conserved among organisms. The complement system forms an important and efficient immune defense mechanism. It consists of plasma proteins that participate in microbial detection, which ultimately results in the production of various molecules with antimicrobial activity. Thioester-containing proteins (TEPs) are a superfamily of secreted effector proteins. In vertebrates, certain TEPs act in

OPEN ACCESS

Edited by:

Larry J. Dishaw,

University of South Florida St.

Petersburg, United States

Reviewed by:

Simon John Clark,

University of Manchester,

United Kingdom

Lubka T. Roumenina,

INSERM UMRS 1138 ,

France

*Correspondence:

loannis Eleftherianos

ioannise@gwu.edu

Specialty section:

This article was submitted to

Molecular Innate Immunity,

a section of the journal

Frontiers in Immunology

Received: 12 May 2017

Accepted: 16 June 2017

Published: 29 June 2017

Citation:

Shokal U and Eleftherianos I (2017) Evolution and Function of Thioester-

Containing Proteins and the

Complement System in the Innate

Immune Response.

Front. Immunol. 8:759.

doi: 10.3389/fimmu.2017.00759 the innate immune response by promoting recruitment of immune cells, phagocytosis, and direct lysis of microbial invaders. Insects are excellent models for dissecting the molecular basis of innate immune recognition and response to a wide range of microbial infections. Impressive progress in recent years has generated crucial information on the role of TEPs in the antibacterial and antiparasite response of the tractable model insect Drosophila melanogaster and the mosquito malaria vector Anopheles gambiae. This knowledge is critical for better understanding the evolution of TEPs and their involvement in the regulation of the host innate immune system.

Keywords: insects, mammals, innate immunity, thioester-containing proteins, complement system, Drosophila, mosquito

\section{INTRODUCTION}

Innate immunity is a fundamental process for early recognition and subsequent induction of proinflammatory responses against invading pathogens (1). Insects are outstanding models for studying innate immune functions and host-pathogen interactions (2-4). Insects activate a variety of innate immune responses depending upon the type of pathogen they encounter. The cell signaling machinery involved in the insect innate immune response is structurally and functionally similar to innate immune pathways in mammals $(5,6)$. Previous and recent research involving infections with bacterial and fungal pathogens has led to the identification and characterization of two distinct immune pathways, the toll pathway [similar to mammalian IL-1/TLR pathway (7)] and the Immune deficiency pathway [Imd, similar to mammalian TNF- $\alpha$ R signaling pathway (8)], which regulate NF- $\kappa B$ transcription factors that control the expression of several antimicrobial peptide (AMP) coding mainly in the fat body tissue (9). In addition, the Janus kinase/signal transducer and activator of transcription (JAK/STAT) and c-Jun N-terminal kinase (JNK) signaling pathways also act in either competing or cooperative modes to modulate the activity of immune effector genes $(10,11)$.

Insects utilize germ line-encoded receptors known as pathogen recognition receptors (PRRs) to identify distinct pathogen-associated molecular patterns (PAMPs) that are either present on the 
surface of microbial pathogens or are released in the host during the infection (12). Insect PRRs are classified into three classessecreted, endocytic, and signaling (13). A special class of signaling PRRs in the fruit fly Drosophila melanogaster is the peptidoglycan recognition proteins (PGRPs) (14). PGRP-SA and PGRP-SD bind to Gram-positive bacteria and activate a protease cascade that induces the toll signaling pathway $(15,16)$. PGRP-LE and PGRP-LC recognize DAP-type peptidoglycan structures present on the Gram-negative bacteria (17). To identify fungal pathogens, PRRs such as Gram-negative binding protein-3 target the $\beta-(1,3)$ glucan structure present on the fungal cell wall (18). Binding of these proteins to their molecular targets results in downstream activation of the NF- $\mathrm{\kappa B}$ signaling pathways Imd and toll (19). In addition to the signaling PRRs, insect genomes also contain secreted recognition molecules such as the thioester-containing proteins (TEPs), named after their active site that functions by forming covalent bonds with specific molecular targets (20). This mini review describes the complement proteins in mammals and the participation of TEPs in the immune response of mosquitoes and flies.

\section{THIOESTER-CONTAINING PROTEINS}

Members of the TEPs family have been recognized in primitive Protostomes and in Deuterostomes, ranging from C. elegans to mammals. TEPs contain a thioester (TE) motif, GCGEQ, which includes a highly unstable covalent bond between the side groups of cysteine and nearby glutamic acid (21). These proteins remain inactive in the native state due to a shielded environment within the protein, but when they encounter elevated temperature, aqueous conditions, or undergo proteolytic activation; the TE bond becomes active for a very short time $(22-24)$. The active TE motif has the ability to bind to nearby accessible hydroxyl and amine groups that are present on all biological surfaces including pathogens (25). TEPs are classified into two subfamilies-complement factors and alpha- 2 macroglobulins $(\alpha-2 \mathrm{Ms})$. Once activated, the complement factors produce a small anaphylatoxin fragment lacking the TE motif and a larger fragment that binds to the target as a result of hydrolysis of the TE bond (20). The small anaphylatoxins act as immunoinflammatory stimulators and chemoattractants that recruit macrophages to the infection site. The larger, covalently bound fragment marks the pathogen as foreign and targets it for lysis or phagocytosis. In contrast, the $\alpha$-2Ms inhibit the protease activity of pathogens via a conformational change that traps the attacking protease after linkage with the TE motif within the protein. This conformational change also exposes the receptor-binding domain of the $\alpha-2 \mathrm{Ms}$ that promotes receptormediated endocytosis for clearance of the pathogen through physical interaction with cell surface receptors (26). Hence, both complement factors and $\alpha-2 \mathrm{Ms}$ serve important functions in recognition as well as clearance of the pathogens from the host. Certain TEPs such as Drosophila TEP6, C5 in higher vertebrates, and ovostatin in mammals, contain a mutated TE motif (27). It has been further suggested that the presence of certain TEPs in the host could be an outcome of different environments, selective pressures, and perhaps gene duplications events $(28,29)$.
Functional characterization of TEPs in model organisms would shed light on their importance and specificity in the host.

\section{COMPLEMENT PROTEINS IN MAMMALS}

The complement system is an important effector that functions at the intersection of innate and adaptive immune responses in mammals. The system includes 50 germ line-encoded, circulating, and membrane-bound proteins. The activation of the complement system triggers a protease cascade that ends in opsonization and/or lysis of the pathogen. In addition to being pro-inflammatory, the complement proteins are also involved in homeostatic processes such as removal of dying cells with exposed danger-associated molecular patterns (DAMPs) that consequently generate a sterile inflammatory reaction $(30,31)$. In certain cases, activation of the complement cascade results in host tissue damage leading to autoimmune and chronic inflammatory diseases (32). Hence, host molecules closely control the activation and regulation of complement system.

The activation of complement system in mammals is regulated through three distinct pathways: the classical pathway, the lectin pathway, and the alternative pathway. Although these pathways have different ligands and receptors, they all converge to produce the same sets of effector molecules (33) (Figure 1A). The initiation of the classical pathway occurs upon binding of the collectin type PRR C1 complex (C1q multimers with inactive serine proteases $\mathrm{C} 1 \mathrm{r}$ and $\mathrm{C} 1 \mathrm{~s}$ ) to an antigen-antibody complex, to PAMPS, or to DAMPs (34-36). When C1q binds to PAMPs, a conformational change occurs in $\mathrm{C} 1 \mathrm{r}$ and $\mathrm{C} 1 \mathrm{~s}$ complex, which results in autocatalytic activation of $\mathrm{C} 1 \mathrm{r}$. The activated $\mathrm{C} 1 \mathrm{r}$ serine proteases then activate the $\mathrm{C} 1 \mathrm{~s}$, which in turn cleave $\mathrm{C} 4$ and $\mathrm{C} 2$ molecules into the small anaphylatoxin $\mathrm{C} 4 \mathrm{a}$ or $\mathrm{C} 2 \mathrm{~b}$ and the larger $\mathrm{C} 4 \mathrm{~b}$ or $\mathrm{C} 2 \mathrm{a}$, respectively. This exposes the activated TE within $\mathrm{C} 4 \mathrm{~b}$, which binds covalently to the pathogen surface and recruits $\mathrm{C} 2 \mathrm{a}$ to form the C4b2a complex. This newly formed complex on the pathogen surface is a C3-convertase that will perpetuate the cascade.

Similar to the classical pathway, the lectin pathway PRRs, either mannan-binding lectin (MBL) or ficolins L/M/H (ficolins$\mathrm{L}$ or ficolins-M or ficolins-H) recognize specific sugars or acetylated moieties on the surfaces of Gram-positive bacteria, Gram-negative bacteria, fungi, protozoans, and viruses (37-39). The lectin pathway PRRs form complex with two MBL-associated serine proteases (MASP)-1 and MASP-2 that are structural homologs of C1r and C1s (40). Thus, MASP-1 and MASP-2 react and cleave $\mathrm{C} 4$ and $\mathrm{C} 2$ molecules to form the same C3-convertase, as described for the classical pathway. In contrast to the classical and lectin pathways, the alternative pathway does not require pathogen recognition proteins for its activation. Instead, it is initiated through spontaneous generation (also called tick-over mechanism) of short-lived $\mathrm{C} 3\left(\mathrm{H}_{2} \mathrm{O}\right)$ by hydrolyzing the TE bond in the $\mathrm{C} 3$ molecule. This short-lived molecule binds to factor B in solution, which causes a conformational change in the structure of factor $\mathrm{B}$. This leads to the cleavage of factor $\mathrm{B}$ into $\mathrm{Ba}$ and $\mathrm{Bb}$ fragments by factor $\mathrm{D}$ forming the $\mathrm{C} 3\left(\mathrm{H}_{2} \mathrm{O}\right) \mathrm{Bb}$ complex, which is the alternative pathway version of a $\mathrm{C} 3$-convertase, also called fluid-phase C3-convertase. 

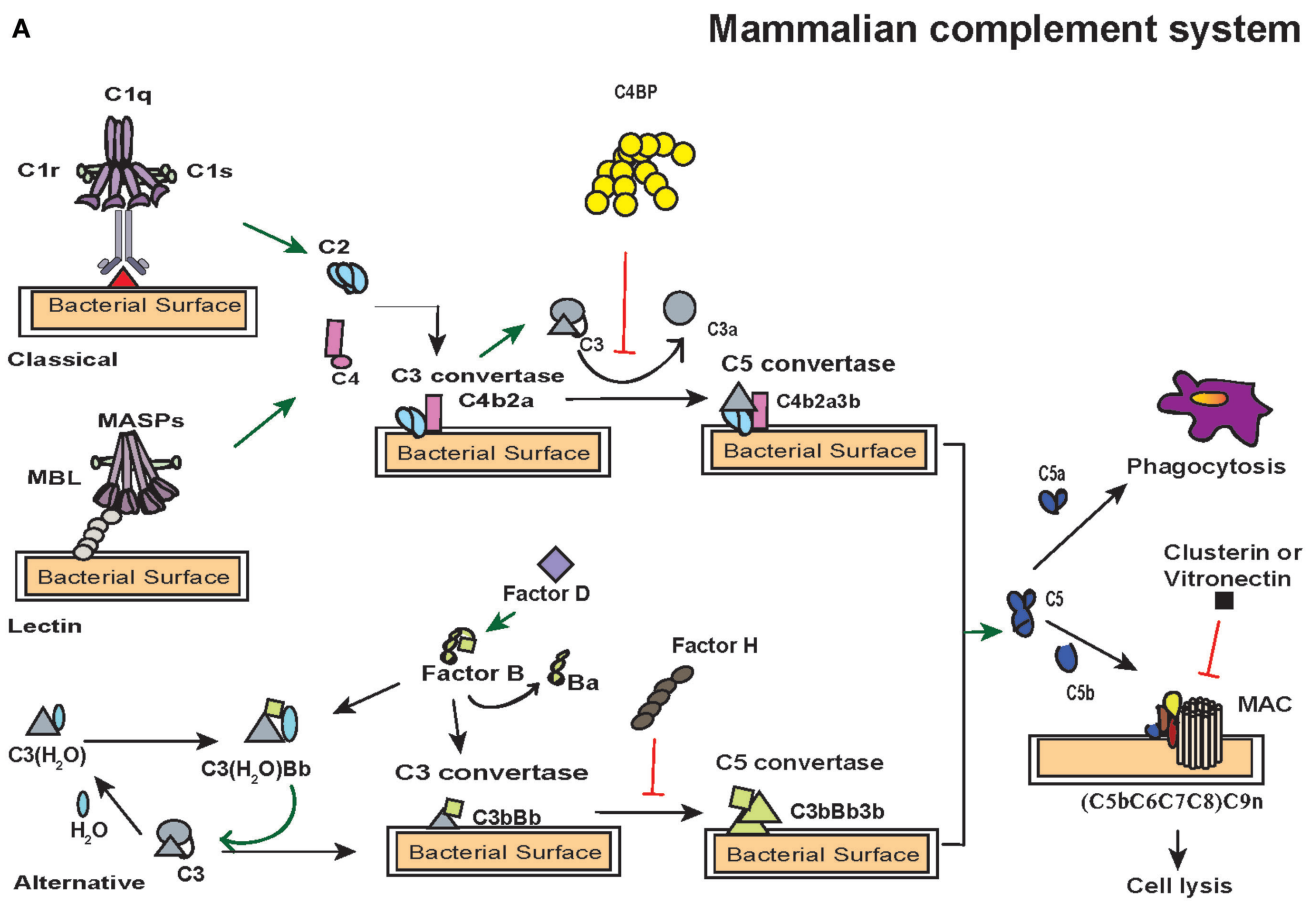

B

Mosquito TEP1 activity

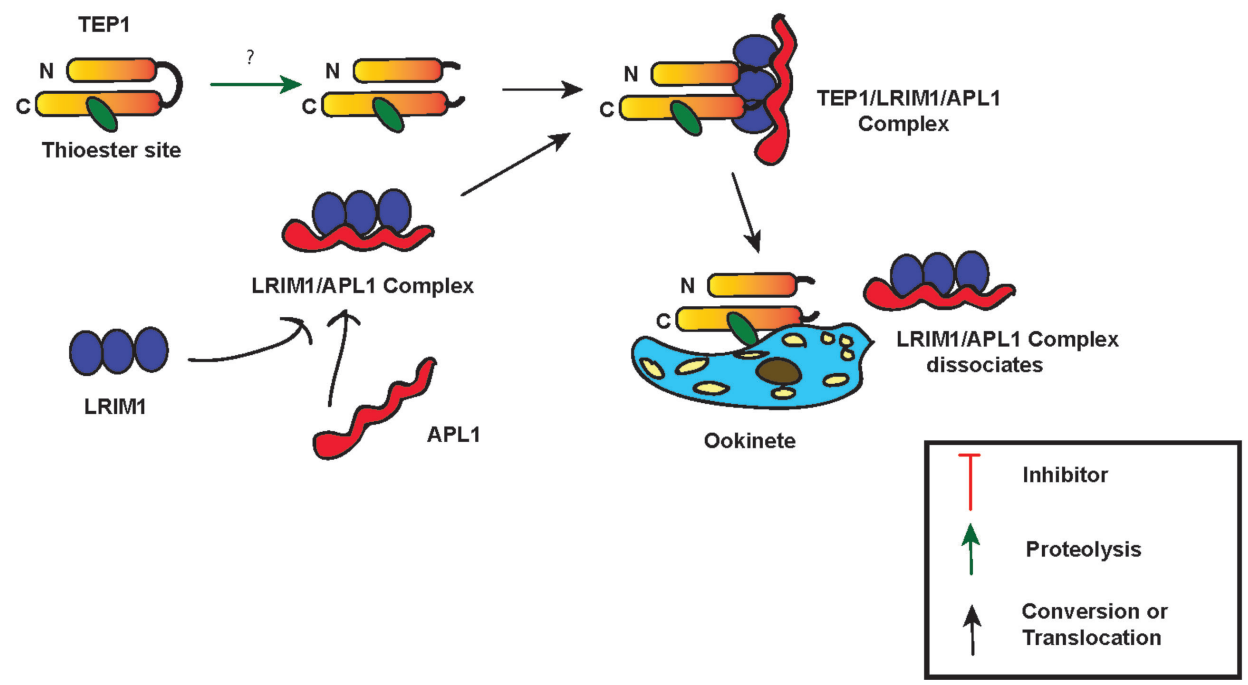

FIGURE 1 | Action of thioester-containing proteins (TEPs) in mammals and mosquitoes. (A) Pattern recognition receptors identify the presence of pathogens. In the classical pathway, C1 complex (specifically C1q) recognizes pathogen-associated molecular patterns or danger-associated molecular patterns either through binding to them directly or through binding of antibodies to the foreign antigen. This activates C1r that subsequently leads to C1s activation. Similarly, in the lectin pathway, binding of mannan-binding lectin (MBL) activates MBL-associated serine protease (MASP-1) and MASP-2. Activation of C1 and/or MBL-MASP complex leads to the cleavage of $\mathrm{C} 4$ and $\mathrm{C} 2$ molecules into $\mathrm{C} 4 \mathrm{a}, \mathrm{C} 4 \mathrm{~b}, \mathrm{C} 2 \mathrm{a}$, and $\mathrm{C} 2 \mathrm{~b}$, subsequently forming $\mathrm{C} 3$ convertase (C4b2a) that binds to the microbial surface. The newly formed C3 convertases cleave C3 into C3b that also binds to the microbial surface. Bound C3b recruits Factor D that activates Factor B, which results in the formation of $\mathrm{C} 3 \mathrm{bBb}$ (C3 convertase of alternative pathway). C3bBb cleaves more $\mathrm{C} 3$ and initiates an amplification loop. Additionally, a fluid-phase convertase could also be formed when water associates with $\mathrm{C} 3$, forming $\mathrm{C} 3\left(\mathrm{H}_{2} \mathrm{O}\right)$. The latter reacts with activated Factor $\mathrm{B}$ and thus maintains a low level of complement activation known as tick-over mechanism. The C3 convertases generated from each pathway bind to C3b forming C5 convertase, which cleaves C5 into C5a and C5b. The latter initiates the formation of membrane attacking membrane by recruiting C6, C7, C8, and C9 complement proteins. Certain molecules such as C4-binding protein, Factor $\mathrm{H}$, vitronectin, and clusterin act as regulators of complement proteins. (B) TEP1 is constitutively activated in the hemolymph by one or more unknown proteases. The proteolytic cleavage produces two fragments TEP1-N and TEP1-C that remain associated with each other. Two leucine-rich repeats (LRRs) proteins, LRIM1 and APL1, maintain the mature form of TEP1. Upon recognition of the parasite, TEP1 dissociates from the LRR proteins by yet an unknown mechanism and binds to the parasite, which ultimately leads to its destruction. Arrows represent inhibition (red), proteolytic cleavage (green), and conversion or translocation of a molecule (black). 
The C3-convertases produced by each of the three pathways generate $\mathrm{C} 5$-convertases upon binding $\mathrm{C} 3 \mathrm{~b}$ to $\mathrm{C} 4 \mathrm{~b} 2 \mathrm{~b}$ in the classical and lectin pathways yielding $\mathrm{C} 4 \mathrm{~b} 2 \mathrm{~b} 3 \mathrm{~b}$. The alternative pathway C3-convertases can cleave many molecules of $\mathrm{C} 3$ into $\mathrm{C} 3 \mathrm{a}$ and $\mathrm{C} 3 \mathrm{~b}$. While most of the $\mathrm{C} 3 \mathrm{~b}$ is inactivated by hydrolysis, a fraction is able to link covalently to the PAMPs through the TE bond and form $\mathrm{C} 3 \mathrm{~b} 2 \mathrm{Bb}$ ( $\mathrm{C} 5$ convertases). The $\mathrm{C} 5$-convertases act on $\mathrm{C} 5$ and cleave it to $\mathrm{C} 5 \mathrm{a}$ and $\mathrm{C} 5 \mathrm{~b}$. $\mathrm{C} 5 \mathrm{a}$ is released as an anaphylatoxin, and $\mathrm{C} 5 \mathrm{~b}$ recruits complement factors $\mathrm{C} 6, \mathrm{C} 7, \mathrm{C} 8$, and C9 that form the membrane-attack complex (MAC) in the cell membrane of the pathogen. While the larger fragment C5b plays a central role in MAC formation, the shorter C5a fragment acts on the endothelial or mast cells and increases the permeability of the blood vessels as well as extravasation of immunoglobulins to the site of inflammation. The activity of C5a causes a septic-shock state called anaphylactic shock and eventually triggers the inflammatory response. Together, these molecules assist in recognition, opsonization, and phagocytosis or lysis of pathogens, and are involved in the activation of adaptive immunity in vertebrates $(41,42)$ (Figure 1A).

The complement factors with TE motifs can also bind selfmolecules containing accessible hydroxyl or amine groups on their surface. Therefore, to avoid false activation of the complement cascade in the absence of foreign entities, several complement regulatory proteins are present in mammals. One of the most potent and well-studied regulatory proteins is complement factor $\mathrm{H}$ that initiates the decay of the $\mathrm{C} 3$-convertase complex by dissociating $\mathrm{Bb}$ from $\mathrm{C} 3 \mathrm{~b}$ (43). Factor $\mathrm{H}$ competes with the $\mathrm{Bb}$ fragment and binds to $\mathrm{C} 3\left(\mathrm{H}_{2} \mathrm{O}\right)$, which results in the dissociation of factor B from the latter. Moreover, it can bind host-specific glycans to prevent complement activation on host surfaces (44). Another regulator is the $\mathrm{C} 4$-binding protein $(\mathrm{C} 4 \mathrm{BP})$ that regulates the classic and lectin pathways with similar activities as factor $\mathrm{H}$ by targeting $\mathrm{C} 4 \mathrm{~b}$ and $\mathrm{C} 2 \mathrm{a}$ (45). C4BP acts as a decay-accelerating factor and dissociates $\mathrm{C} 2 \mathrm{a}$ from the $\mathrm{C} 3$-convertases. While these regulators control the formation of $\mathrm{C} 3$-convertase, other complement regulators such as clusterin and vitronectin inhibit MAC assembly or C9 insertion into membranes after the formation of C3 convertase complex and activation of the terminal pathway $(46,47)$.

Although the complement system is extremely efficient in fighting and clearing pathogenic infections, certain bacterial and viral pathogens can evade this immune response $(48,49)$. They achieve this by escaping the complement action through binding to the complement inhibitors, which target active complement factors that interfere with MAC complex formation and mimic host surfaces (50-52).

\section{TEPS IN INSECTS}

Phylogenetic analysis of TEP-coding genes in dipteran insects, other invertebrates, and vertebrate animals has classified them into three subfamilies including complement factors, $\alpha-2 \mathrm{Ms}$, and insect TEPs (20) (Figure 2A). The complement factor subgroup containing C3, C4, and C5 proteins is the most fast-evolved TEP subfamily. On the other hand, the $\alpha-2 \mathrm{Ms}$ are present in a larger group of animals other than the two subfamilies, which suggests their slow evolution due to several functional constraints on the structure of these inhibitors (53). Insect TEPs are highly diverged as well as unstable, and they are more related to the $\alpha-2 \mathrm{M}$ family than to the TE complement factor group (20) (Figure 2A). The presence of multiple TEP homologs in mosquitoes relative to those in Drosophila indicates that different adaptations between these insects have led to gene duplication and the generation of more homologs (54). It is currently unknown whether regulators of TEPs, such as homologs of human C4BP or Factor $\mathrm{H}$, in insects exist. Interestingly, mosquitoes can capture Factor $\mathrm{H}$ from ingested human blood to escape the deleterious effects of the complement activation system (55). Although there is high structural and functional homology between TEPs and complement proteins, it is unclear whether insect TEPs possess a mechanism of action similar to $\mathrm{C} 3$ tick over. Here, we summarize TEPs in mosquitoes and fruit flies.

\section{Mosquito TEPs}

Genome sequencing of two mosquito species, Anopheles gambiae and Aedes aegypti, has contributed toward understanding several molecular mechanisms involved in host immunity. Various components of the complement pathway, specifically, complementlike proteins have been identified in the two genomes. The A. gambiae genome contains 19 TEP gene homologs (AgTep 1-19), of which four pairs show haplotypic features (AgTep1-AgTep16, AgTep5-AgTep17, AgTep6-AgTep18, and AgTep7-AgTep19) and hence, represent polymorphic variations rather than distinct genes $(54,56)$. There are eight Tep genes in A. aegypti (AeTEP 1-8) encoding TEP proteins that share $21-39 \%$ amino acid similarity to $\operatorname{AgTEP} 1(57,58)$. In addition, the mosquito TEPs share structural and functional similarities with mammalian $\alpha$-2Ms $(29,59)$.

A key immune gene identified through functional studies in A. gambiae was AgTep1. AgTEP1 is a constitutively secreted hemolymph protein with a size of $165 \mathrm{kDa}$ (TEP1-full) and its cleavage results in the formation of an $80 \mathrm{kDa}$ active fragment (TEP1-cut) (60). While the N-terminal region of AgTEP1 has a hydrophobic signal peptide-like segment as well as a canonical TE motif plus a catalytic histidine residue that is positioned 100 amino acids downstream, the $\mathrm{C}$-terminal region has a cysteine signature. The TEP1-cut circulates in the hemolymph in association with two leucine-rich repeats (LRRs) proteins, LRIM1 and APL1C $(61,62)$. These two LRR proteins act as TEPs regulators and promote pathogen recognition as well as their destruction (Figure 1B).

Several studies have shown the functional importance of AgTEP1 in various processes such as recognition, opsonization, and phagocytosis of certain bacteria. In vitro and in vivo studies have shown that bacteria are phagocytosed when the C-terminal part of AgTEP1, also called AgTEP1-cut, binds to bacteria (63). Moreover, knockdown of AgTep1 or culturing of hemocytes with methylamine-treated conditioned medium (prevents autocatalytic fragmentation of the full-length protein into smaller $80 \mathrm{kDa}$ cut fragment) reduced the efficiency of phagocytosis of Gramnegative bacteria by $50-75 \%$ (63). Another study also showed decrease in phagocytosis of Escherichia coli and Staphylococcus aureus after the depletion of AgTep1 expression (62). Thus, 
A

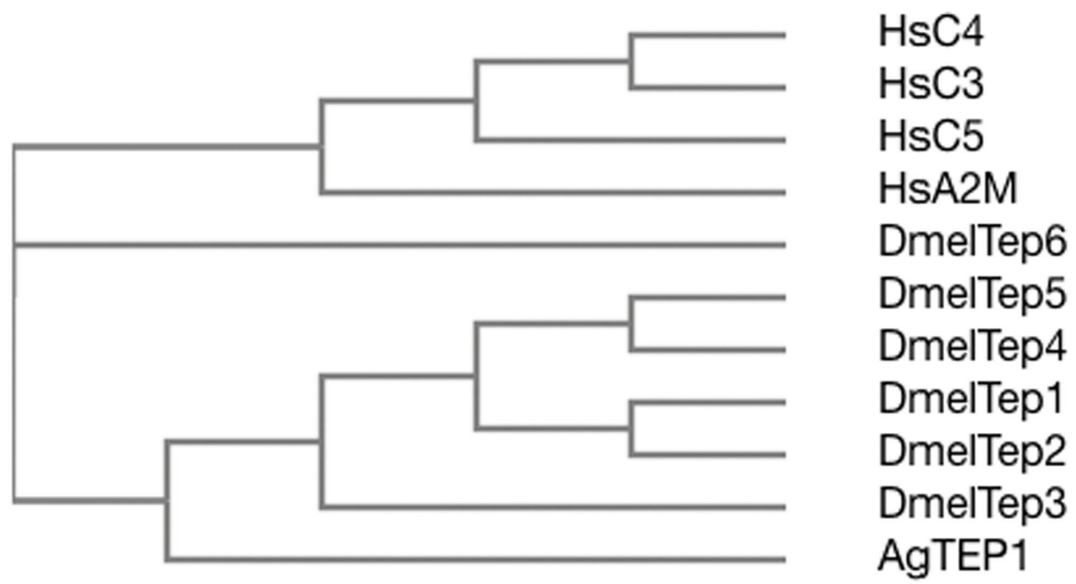

B

Humans
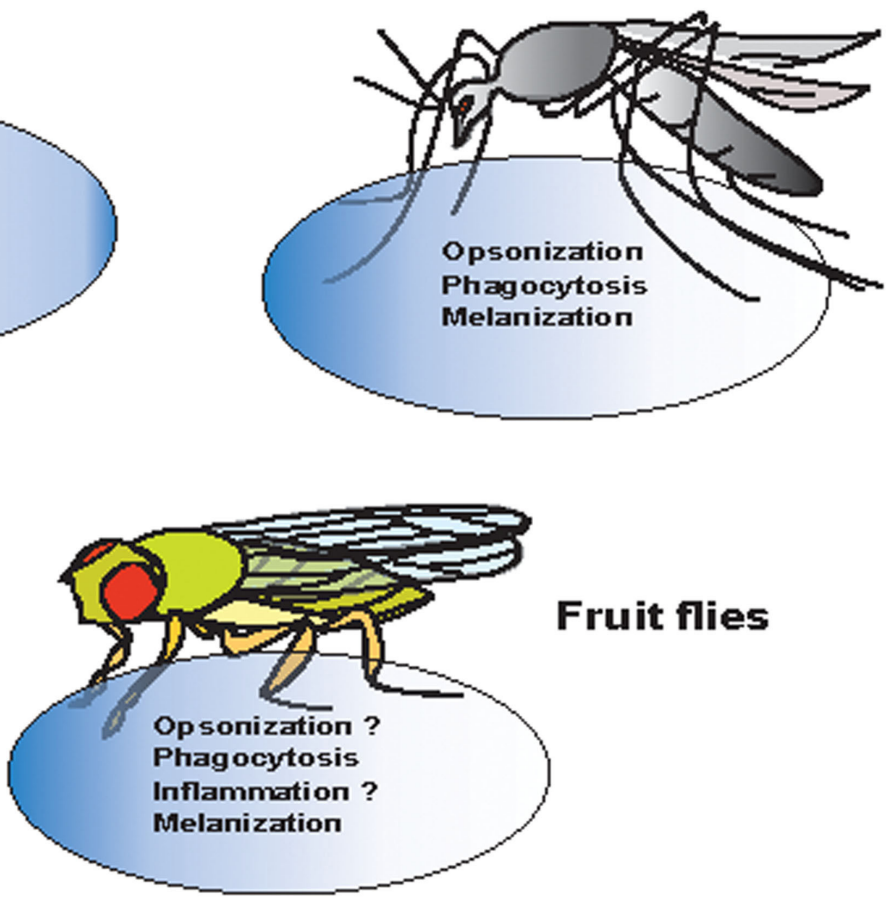

FIGURE 2 | Evolution and conserved function of thioester-containing proteins (TEPs) in mammals, mosquitoes, and fruit flies. (A) Phylogenetic tree showing the evolution and similarity between different complement factors, alpha-2 macroglobulin in Homo sapiens (Hs), and TEPs in Anopheles gambiae (Ae) and Drosophila melanogaster (Dm), generated through Clustal-Omega. (B) Complement proteins are involved in immune functions such as opsonization, phagocytosis, cell lysis of pathogens, and act as pro-inflammatory molecules. Similarly in mosquitoes, TEP molecules participate in the opsonization, phagocytosis, and melanization of bacterial pathogens and Plasmodium parasites. However, with the exception of phagocytosis and melanization, immune anti-pathogen activities of TEPs are yet to be identified in the fly.

AgTEP1 acts as an opsonin and marks targeted bacteria for phagocytosis (Figure 2B).

The complement C3-like protein, AgTEP1, is an important molecule in inducing an immune response against Plasmodium berghei. The protein binds to the surface of the parasite and triggers its encapsulation by hemocytes, which leads to parasite death. Two divergent alleles of AgTep1-AgTep1r and AgTep1s are reported (64). While AgTep1s is present in most mosquito populations making them susceptible to $P$. berghei infection, the allele AgTep1r confers high resistance to the same parasite (65). Moreover, silencing of AgTep1 also inhibits parasitic lysis and actin polymerization (66). The regulatory molecules LRIM1 and 
APL1 are also required for binding of AgTEP1 to the parasites (61). The LRIM1 and APL1 complex not only interacts with AgTEP1 but also interferes with three other TEP proteins, including AgTEP3 (64). Silencing the two genes encoding the LRR proteins results in the conversion of refractory strains into susceptible strains $(61,63)$.

Although several functional studies have been performed on AgTEPs, only a few studies have characterized the specific function of $A$. aegypti TEPs. The genes AeTep1-AeTep5 are all constitutively expressed throughout the body of adult mosquitoes. A study on $A$. aegypti has shown a twofold to threefold increase in West Nile virus load after silencing the AeTep1 and AeTep2 genes whereas overexpression of AeTep1 and AeTep3 resulted in a decrease in viral load (58). Thus, AeTEPs has an important function in the mosquito host defense by limiting viral infection.

The mosquito TEPs have been found to possess conserved function similar to complement factors by binding to bacteria or Plasmodium parasites, which results in the phagocytosis of bacteria as well as melanization and lysis of the parasites, respectively (59) (Figure 2B). Future studies will focus on investigating the binding specificity of mosquito TEPs and how the binding process leads to parasite lysis at the molecular level.

\section{Fruit Fly TEPs}

In insects, TEPs were first discovered in Drosophila melanogaster (67). While there is a plethora of information on mosquito TEPs, there are only few studies on the immune function of TEPs in Drosophila. The D. melanogaster genome contains six TEP homologs (68). TEP1-4 contain a conserved TE motif. Tep5 may represent a pseudogene as it is found in the genomic sequences but is not expressed (13). Drosophila TEPs have a highly conserved region of 30 amino acids that compose the N-terminal of the TE motif, a cysteine signature tail, which is similar to Anopheles TEPs. They also have a 60 amino acid hypervariable region, which is structurally similar to mammalian bait region of $\alpha-2 \mathrm{Ms}$ as well as to the anaphylatoxin domain in vertebrate C3b (67). TEP6 is the only TEP that lacks a functional TE motif, exhibiting a serine instead of cysteine residue. Of the six Tep genes, only Tep 2 shows alternative splicing in exon 5 producing five different isoforms. The alternative splicing occurs in the exon region that codes for the hypervariable domain of the TEP2 protein. The alternative splicing may aid in increasing the inhibitor proteases repertoire and augmented diversity of recognition receptors. Flies may have evolved a strategy to encounter distinct pathogens that is analogous to VDJ diversity generated by adaptive immunity in higher vertebrates (69).

Drosophila melanogaster Teps are upregulated in different tissues and participate in immune response and developmental processes. Teps are expressed in larval hemocytes, fat body, and in the gut barrier epithelia, whereas, in the case of adults, Teps are expressed in the fat body of the head, spermatozoa, and midgut epithelia in the absence of infection (70). Upon bacterial challenge, Tep1, Tep2, and Tep4 are upregulated in D. melanogaster larvae, whereas only Tep1, Тер2, Тер4, and Тер6 are upregulated in adults in response to certain bacterial, fungal, or parasitoid infection (67, 70-72). Additionally, Тep2 and Tep3 are upregulated against parasitic infections with the nematode Heterorhabditis bacteriophora that contains the mutualistic bacteria Photorhabdus luminescens (73). Loss-of-function tep 2 and tep 4 mutants are susceptible to Pseudomonas ginigivalis infection whereas loss-of-function tep 3 mutants are susceptible to $H$. bacteriophora infection $(74,75)$. Another study reported that tep1-4 mutant flies were slightly resistant to bacterial infection in comparison to wild-type flies (69). Although these studies reported the involvement of fly TEPs in the antibacterial and antiparasitic immune response, the mechanism of TEPs action was not clarified. More recently, it was shown that TEP2, TEP4, and TEP6 has an important regulatory role in the innate immune response of $D$. melanogaster adult flies against the pathogenic bacteria Photorhabdus $(72,76)$. Tep2, Tep4, and Tep 6 are transcriptionally upregulated in response to $P$. lumi$n e s c e n s$ and $P$. asymbiotica infection. Moreover, transcriptional activation of these genes influences the activation of toll, Imd, JAK/STAT, and JNK signaling and results in differential expression of AMP and stress coding genes. Tep2 and Tep4 upregulation also decreases phenoloxidase activity and the melanization response during the early stages of Photorhabdus infection. As a result, these effects promote the survival of flies upon infection with pathogenic Photorhabdus. This is the first evidence of the involvement of a TEP in the fly antibacterial immune system.

In vitro, D. melanogaster TEP2, TEP4, and TEP6 (MCR or macroglobulin-complement related) promote phagocytosis of certain Gram-negative bacteria and fungal pathogens (77). The rate of phagocytosis in D. melanogaster S2 cells incubated with Candida albicans decreases upon Mcr silencing because the MCR protein binds specifically to the fungal surface. Moreover, the rate of $E$. coli and $S$. aureus phagocytosis is reduced after silencing Tep 2 and Tep 4 genes. In addition, inactivation of Tep 2 and Tep6 significantly impairs the expression of Eater gene in adult flies suggesting that TEP2 and TEP6 participate in the phagocytic response against Photorhabdus bacteria (72) (Figure 2B). This suggests that different TEP molecules are involved in the immune response and probably recognition of different pathogens. It has been suggested previously that JAK/STAT and toll pathways regulate the expression of TEP1, but the mechanisms are poorly understood $(67,78)$.

\section{CONCLUDING REMARKS AND FUTURE PROSPECTS}

Recent efforts have mostly focused on understanding the molecular and genetic mechanisms that regulate the participation of TEPs in interfering with the transmission of eukaryotic parasites and activating innate immune responses against pathogenic infections in insects $(76,79-82)$. Future studies could potentially examine the tissue-specific patterns of induction of insect Tep genes upon infection with different pathogenic and non-pathogenic microorganisms. Tissue-specific profiling of Tep gene expression would possible denote their specificity toward certain microbial infections. For example, the upregulation of Tep genes in the fat body, gut, or hemocytes upon microbial challenge would indicate their involvement in the insect humoral and/or cellular immune response to microbial invaders. Indeed, complement proteins 
are involved in the activation the humoral immune response in invertebrates and vertebrates. In mammals, complement factors are involved in the regulation of humoral immune responses (83). In insects, complement-related factors participate in the upregulation of AMPs against flavivirus infection in the mosquito, $A$. aegypti (84). Recently, it has been proposed that macrocapsules loaded with $\alpha$-2Ms enhance certain human leukocyte functions, such as the recruitment of leukocytes to the site of inflammation and phagocytosis (85). Although TEP1 is involved in opsonization and phagocytosis of certain bacteria in mosquitoes $(20,26)$, other TEP molecules, might also participate directly or indirectly in insect cellular immune processes.

In addition in mammals, there is an intricate cross talk between the complement system and the coagulation cascade (86). Within certain hours of pathogenic infection, both of these systems are activated through the activity of serine proteases (87). Likewise, the coagulation system and the phenoloxidase cascade are linked in insects (88). It has been shown that A. gambiae TEP1 is essential in the process of melanization of Plasmodium parasites (6), and phenoloxidase activity as well as the melanization response are affected in Drosophila flies inactivated for tep2 and tep 4 genes when responding to the pathogen Photorhabdus $(72,76)$ (Figure 2B). Future research could concentrate on the identification of the molecular components that facilitate the interaction between complement and coagulation systems in vertebrates and invertebrates.

\section{REFERENCES}

1. Medzhitov R, Janeway C Jr. Innate immunity. N Engl J Med (2000) 343(5):338-44. doi:10.1056/NEJM200008033430506

2. Limmer S, Quintin J, Hetru C, Ferrandon D. Virulence on the fly: Drosophila melanogaster as a model genetic organism to decipher host-pathogen interactions. Curr Drug Targets (2011) 12(7):978-99. doi: $10.2174 / 138945011795677818$

3. Buchon N, Silverman N, Cherry S. Immunity in Drosophila melanogaster from microbial recognition to whole-organism physiology. Nat Rev Immunol (2014) 14(12):796-810. doi:10.1038/nri3763

4. Wojda I. Immunity of the greater wax moth Galleria mellonella. Insect Sci (2017) 24(3):342-57. doi:10.1111/1744-7917.12325

5. Vilmos P, Kurucz E. Insect immunity: evolutionary roots of the mammalian innate immune system. Immunol Lett (1998) 62(2):59-66. doi:10.1016/ S0165-2478(98)00023-6

6. Viljakainen L. Evolutionary genetics of insect innate immunity. Brief Funct Genomics (2015) 14(6):407-12. doi:10.1093/bfgp/elv002

7. Lindsay SA, Wasserman SA. Conventional and non-conventional Drosophila toll signaling. Dev Comp Immunol (2014) 42(1):16-24. doi:10.1016/j. dci.2013.04.011

8. Myllymäki H, Valanne S, Rämet M. The Drosophila IMD signaling pathway. J Immunol (2014) 192(8):3455-62. doi:10.4049/jimmunol.1303309

9. Yi HY, Chowdhury M, Huang YD, Yu XQ. Insect antimicrobial peptides and their applications. Appl Microbiol Biotechnol (2014) 98(13):5807-22. doi:10.1007/s00253-014-5792-6

10. Zeidler MP, Bausek N. The Drosophila JAK-STAT pathway. JAKSTAT (2013) 2(3):e25353. doi:10.4161/jkst.25353

11. Zhou YY, Li Y, Jiang WQ, Zhou LF. MAPK/JNK signalling: a potential autophagy regulation pathway. Biosci Rep (2015) 35(3):e00199. doi:10.1042/ BSR20140141

12. Pal S, Wu LP. Pattern recognition receptors in the fly: lessons we can learn from the Drosophila melanogaster immune system. Fly (2009) 3(2):121-9. doi:10.4161/fly.8827
Complement proteins are involved in the inflammation process and programmed cell death in vertebrates $(89,90)$. The presence of complement serves a protective function in vertebrates, but complement activation can also be deleterious for the host (91). Deletion in C5a confers resistance and reduced bacteremia shock in mice in response to Gram-negative bacterial infection (92). Identification of TEPs in insects with function analogous to $\mathrm{C} 5 \mathrm{a}$ in mammals or relevance to pathophysiological defects in the host offers an exciting and challenging area of future research. In conclusion, future studies on elucidating the molecular mechanisms of interaction of TEPs with specific host physiological processes will undoubtedly shed light on their exact anti-pathogen immune function as well as their evolution in the animal kingdom.

\section{AUTHOR CONTRIBUTIONS}

US wrote the paper and IE revised it.

\section{FUNDING}

The Eleftherianos laboratory is funded by grants from the National Institutes of Health-National Institute of Allergy and Infectious Diseases (1R01AI110675, 1R56AI110675-01, and 1R21AI109517) and the Columbian College of Arts and Sciences at George Washington University.

13. Stokes BA, Yadav S, Shokal U, Smith LC, Eleftherianos I. Bacterial and fungal pattern recognition receptors in homologous innate signaling pathways of insects and mammals. Front Microbiol (2015) 6:19. doi:10.3389/ fmicb.2015.00019

14. Kurata S. Peptidoglycan recognition proteins in Drosophila immunity. Dev Comp Immunol (2014) 42(1):36-41. doi:10.1016/j.dci.2013.06.006

15. Werner T, Liu G, Kang D, Ekengren S, Steiner H, Hultmark D. A family of peptidoglycan recognition proteins in the fruit fly Drosophila melanogaster. Proc Natl Acad Sci U S A (2000) 97:13772-7. doi:10.1073/pnas.97.25.13772

16. Michel T, Reichhart JM, Hoffmann JA, Royet J. Drosophila toll is activated by Gram-positive bacteria through a circulating peptidoglycan recognition protein. Nature (2001) 414:756-9. doi:10.1038/414756a

17. Takehana A, Yano T, Mita S, Kotani A, Oshima Y, Kurata S. Peptidoglycan recognition protein (PGRP)-LE and PGRP-LC act synergistically in Drosophila immunity. EMBO J (2004) 23:4690-700. doi:10.1038/sj.emboj. 7600466

18. Gottar M, Gobert V, Matskevich AA, Reichhart JM, Wang C, Butt TM, et al. Dual detection of fungal infections in Drosophila via recognition of glucans and sensing of virulence factors. Cell (2006) 127:1425-37. doi:10.1016/j. cell.2006.10.046

19. Ferrandon D, Imler JL, Hetru C, Hoffmann JA. The Drosophila systemic immune response: sensing and signaling during bacterial and fungal infections. Nat Rev Immunol (2007) 7(11):862-74. doi:10.1038/nri2194

20. Blandin S, Levashina EA. Thioester-containing proteins and insect immunity. Mol Immunol (2004) 40(12):903-8. doi:10.1016/j.molimm.2003.10.010

21. Law SK, Dodds AW. The internal thioester and the covalent binding properties of the complement proteins C3 and C4. Protein Sci (1997) 6(2):263-74. doi:10.1002/pro.5560060201

22. Salvesen GS, Sayers CA, Barrett AJ. Further characterization of the covalent linking reaction of alpha 2-macroglobulin. Biochem J (1981) 195(2):453-61. doi:10.1042/bj1950453

23. Sim RB, Sim E. Autolytic fragmentation of complement components C3 and C4 under denaturing conditions, a property shared with alpha 2-macroglobulin. Biochem J (1981) 193(1):129-41. doi:10.1042/bj1930129 
24. Sahu A, Kozel TR, Pangburn MK. Specificity of the thioester-containing reactive site of human C3 and its significance to complement activation. Biochem $J$ (1994) 302(2):429-36. doi:10.1042/bj3020429

25. Dodds AW, Ren XD, Willis AC, Law SK. The reaction mechanism of the internal thioester in the human complement component C4. Nature (1996) 379(6561):177-9. doi:10.1038/379177a0

26. Blandin SA, Marois E, Levashina EA. Antimalarial responses in Anopheles gambiae: from a complement-like protein to a complement-like pathway. Cell Host Microbe (2008) 3(6):364-74. doi:10.1016/j.chom.2008.05.007

27. Janssen BJ, Huizinga EG, Raaijmakers HC, Roos A, Daha MR, NilssonEkdahl K, et al. Structures of complement component C3 provide insights into the function and evolution of immunity. Nature (2005) 437(7058):505-11. doi:10.1038/nature04005

28. Nonaka M, Yoshizaki F. Primitive complement system of invertebrates. Immunol Rev (2004) 198:203-15. doi:10.1111/j.0105-2896.2004.00118.x

29. Nonaka M, Kimura A. Genomic view of the evolution of the complement system. Immunogenetics (2006) 58(9):701-13. doi:10.1007/s00251-006-0142-1

30. Rock KL, Latz E, Ontiveros F, Kono H. The sterile inflammatory response. Ann RevImmunol (2010) 28:321-42. doi:10.1146/annurev-immunol-030409101311

31. Carroll MC, Isenman DE. Regulation of humoral immunity by complement. Immunity (2012) 37(2):199-207. doi:10.1016/j.immuni.2012.08.002

32. Dunkelberger JR, Song WC. Complement and its role in innate and adaptive immune responses. Cell Res (2010) 20(1):34-50. doi:10.1038/ cr.2009.139

33. Dodds AW. Which came first, the lectin/classical pathway or the alternative pathway of complement? Immunobiology (2002) 205(4-5):340-54. doi:10.1078/0171-2985-00137

34. Arlaud GJ, Gaboriaud C, Thielens NM, Rossi V, Bersch B, Hernandez JF, et al. Structural biology of C1: dissection of a complex molecular machinery. Immunol Rev (2001) 180:136-45. doi:10.1034/j.1600-065X.2001.1800112.x

35. Nayak A, Pednekar L, Reid KB, Kishore U. Complement and non-complement activating functions of C1q: a prototypical innate immune molecule. Innate Immun (2012) 18(2):350-63. doi:10.1177/1753425910396252

36. Ramirez-Ortiz ZG, Pendergraft WF III, Prasad A, Byrne MH, Iram T, Blanchette CJ, et al. The scavenger receptor SCARF1 mediates the clearance of apoptotic cells and prevents autoimmunity. Nat Immunol (2013) 14(9):917-26. doi:10.1038/ni.2670

37. Degn SE, Thiel S. Humoral pattern recognition and the complement system. Scand J Immunol (2013) 78(2):181-93. doi:10.1111/sji.12070

38. Kjaer TR, Thiel S, Andersen GR. Toward a structure-based comprehension of the lectin pathway of complement. Mol Immunol (2013) 56(4):413-22. doi:10.1016/j.molimm.2013.05.007

39. Matsushita M. Ficolins in complement activation. Mol Immunol (2013) 55(1):22-6. doi:10.1016/j.molimm.2012.08.017

40. Bajic G, Degn SE, Thiel S, Andersen GR. Complement activation, regulation, and molecular basis for complement-related diseases. EMBO J (2015) 34(22):2735-57. doi:10.15252/embj.201591881

41. Carroll MC. The complement system in regulation of adaptive immunity. Nat Immunol (2004) 5(10):981-6. doi:10.1038/ni1113

42. Morgan BP, Marchbank KJ, Longhi MP, Harris CL, Gallimore AM. Complement: central to innate immunity and bridging to adaptive responses. Immunol Lett (2005) 97(2):171-9. doi:10.1016/j.imlet.2004.11.010

43. Ferreira VP, Pangburn MK, Cortes C. Complement control protein factor $\mathrm{H}$ : the good, the bad, and the inadequate. Mol Immunol (2010) 47(13):2187-97. doi:10.1016/j.molimm.2010.05.007

44. Makou E, Herbert AP, Barlow PN. Functional anatomy of complement factor H. Biochemistry (2013) 52(23):3949-62. doi:10.1021/bi4003452

45. Noris M, Remuzzi G. Overview of complement activation and regulation. Semin Nephrol (2013) 33(6):479-92. doi:10.1016/j.semnephrol.2013.08.001

46. Tschopp J, French LE. Clusterin: modulation of complement function. Clin Exp Immunol (1994) 97(Suppl 2):11-4. doi:10.1111/j.1365-2249.1994.tb06256.x

47. Koch-Brandt C, Morgans C. Clusterin: a role in cell survival in the face of apoptosis? Prog Mol Subcell Biol (1996) 16:130-49. doi:10.1007/978-3642-79850-4_8

48. Serruto D, Rappuoli R, Scarselli M, Gros P, van Strijp JA. Molecular mechanisms of complement evasion: learning from staphylococci and meningococci. Nat Rev Microbiol (2010) 8:393-9. doi:10.1038/nrmicro2366
49. Rettig TA, Harbin JN, Harrington A, Dohmen L, Fleming SD. Evasion and interactions of the humoral innate immune response in pathogen invasion, autoimmune disease, and cancer. Clin Immunol (2015) 160:244-54. doi:10.1016/j.clim.2015.06.012

50. Zipfel PF, Würzner R, Skerka C. Complement evasion of pathogens: common strategies are shared by diverse organisms. Mol Immunol (2007) 44(16): 3850-7. doi:10.1016/j.molimm.2007.06.149

51. Zipfel PF, Hallström T, Riesbeck K. Human complement control and complement evasion by pathogenic microbes - tipping the balance. Mol Immunol (2013) 56(3):152-60. doi:10.1016/j.molimm.2013.05.222

52. Hovingh ES, van den Broek B, Jongerius I. Hijacking complement regulatory proteins for bacterial immune evasion. Front Microbiol (2016) 7:2004. doi:10.3389/fmicb.2016.02004

53. Dodds AW, Law SK. The phylogeny and evolution of the thioester bond-containing proteins C3, C4 and alpha 2-macroglobulin. Immunol Rev (1998) 166:15-26. doi:10.1111/j.1600-065X.1998.tb01249.x

54. Christophides GK, Zdobnov E, Barillas-Mury C, Birney E, Blandin S, Blass C, et al. Immunity-related genes and gene families in Anopheles gambiae. Science (2002) 298(5591):159-65. doi:10.1126/science.1077136

55. Khattab A, Barroso M, Miettinen T, Meri S. Anopheles midgut epithelium evades human complement activity by capturing factor $\mathrm{H}$ from the blood meal. PLoS Negl Trop Dis (2015) 9:e0003513. doi:10.1371/journal. pntd.0003513

56. Christophides GK, Vlachou D, Kafatos FC. Comparative and functional genomics of the innate immune system in the malaria vector Anopheles gambiae. Immunol Rev (2004) 198:127-48. doi:10.1111/j.0105-2896.2004.0127.x

57. Waterhouse RM, Kriventseva EV, Meister S, Xi Z, Alvarez KS, Bartholomay LC, et al. Evolutionary dynamics of immune-related genes and pathways in disease-vector mosquitoes. Science (2007) 316(5832):1738-43. doi:10.1126/science.1139862

58. Cheng G, Liu L, Wang P, Zhang Y, Zhao YO, Colpitts TM, et al. An in vivo transfection approach elucidates a role for Aedes aegypti thioester-containing proteins in flaviviral infection. PLoS One (2011) 6:e22786. doi:10.1371/ journal.pone.0022786

59. Williams M, Baxter R. The structure and function of thioester-containing proteins in arthropods. Biophys Rev (2014) 6(3):261-72. doi:10.1007/s12551014-0142-6

60. Levashina EA, Moita LF, Blandin S, Vriend G, Lagueux M, Kafatos FC. Conserved role of a complement-like protein in phagocytosis revealed by dsRNA knockout in cultured cells of the mosquito, Anopheles gambiae. Cell (2001) 104(5):709-18. doi:10.1016/S0092-8674(01)00267-7

61. Fraiture M, Baxter RH, Steinert S, Chelliah Y, Frolet C, Quispe-Tintaya W, et al. Two mosquito LRR proteins function as complement control factors in the TEP1-mediated killing of Plasmodium. Cell Host Microbe (2009) 5(3):273-84. doi:10.1016/j.chom.2009.01.005

62. Moita LF, Wang-Sattler R, Michel K, Zimmermann T, Blandin S, Levashina EA, et al. In vivo identification of novel regulators and conserved pathways of phagocytosis in A. gambiae. Immunity (2005) 23(1):65-73. doi:10.1016/j. immuni.2005.05.006

63. Povelones M, Waterhouse RM, Kafatos FC, Christophides GK. Leucine-rich repeat protein complex activates mosquito complement in defense against Plasmodium parasites. Science (2009) 324(5924):258-61. doi:10.1126/science. 1171400

64. Blandin S, Shiao SH, Moita LF, Janse CJ, Waters AP, Kafatos FC, et al. Complement-like protein TEP1 is a determinant of vectorial capacity in the malaria vector Anopheles gambiae. Cell (2004) 116(5):661-70. doi:10.1016/ S0092-8674(04)00173-4

65. Blandin SA, Wang-Sattler R, Lamacchia M, Gagneur J, Lycett G, Ning Y, et al. Dissecting the genetic basis of resistance to malaria parasites in Anopheles gambiae. Science (2009) 326(5949):147-50. doi:10.1126/science.1175241

66. Shiao SH, Whitten MM, Zachary D, Hoffmann JA, Levashina EA. Fz2 and cdc42 mediate melanization and actin polymerization but are dispensable for Plasmodium killing in the mosquito midgut. PLoS Pathog (2006) 2:e133. doi:10.1371/journal.ppat.0020133

67. Lagueux M, Perrodou E, Levashina EA, Capovilla M, Hoffmann JA. Constitutive expression of a complement-like protein in toll and JAK gain-offunction mutants of Drosophila. Proc Natl Acad Sci U S A (2000) 97:11427-32. doi:10.1073/pnas.97.21.11427 
68. Adams MD, Celniker SE, Holt RA, Evans CA, Gocayne JD, Amanatides PG, et al. The genome sequence of Drosophila melanogaster. Science (2000) 287(5461):2185-95. doi:10.1126/science.287.5461.2185

69. Bou Aoun R, Hetru C, Troxler L, Doucet D, Ferrandon D, Matt N. Analysis of thioester-containing proteins during the innate immune response of Drosophila melanogaster. J Innate Immun (2011) 3(1):52-64. doi:10.1159/ 000321554

70. Clark AG, Eisen MB, Smith DR, Bergman CM, Oliver B, Markow TA, et al. Evolution of genes and genomes on the Drosophila phylogeny. Nature (2007) 450(7167):203-18. doi:10.1038/nature06341

71. Wertheim B, Kraaijeveld AR, Schuster E, Blanc E, Hopkins M, Pletchers SD, et al. Genome-wide gene expression in response to parasitoid attack in Drosophila. Genome Biol (2005) 6:R94. doi:10.1186/gb-2005-6-11-r94

72. Shokal U, Kopydlowski H, Eleftherianos I. The distinct function of Tep2 and Tep6 in the immune defense of Drosophila melanogaster against the pathogen Photorhabdus. Virulence (2017) 1-15. doi:10.1080/21505594.20 17.1330240

73. Castillo JC, Creasy T, Kumari P, Shetty A, Shokal U, Tallon LJ, et al. Drosophila anti-nematode and antibacterial immune regulators revealed by RNA-Seq. BMC Genomics (2015) 16:519. doi:10.1186/s12864-015-1690-2

74. Igboin CO, Tordoff KP, Moeschberger ML, Griffen AL, Leys EJ. Porphyromonas gingivalis-host interactions in a Drosophila melanogaster model. Infect Immun (2011) 79:449-58. doi:10.1128/IAI.00785-10

75. Arefin B, Kucerova L, Dobes P, Markus R, Strnad H, Wang Z, et al. Genomewide transcriptional analysis of Drosophila larvae infected by entomopathogenic nematodes shows involvement of complement, recognition and extracellular matrix proteins. J Innate Immun (2014) 6:192-204. doi:10.1159/ 000353734

76. Shokal U, Eleftherianos I. Thioester-containing protein-4 regulates the Drosophila immune signaling and function against the pathogen Photorhabdus. J Innate Immun (2017) 9(1):83-93. doi:10.1159/000450610

77. Stroschein-Stevenson SL, Foley E, O'Farrell PH, Johnson AD. Identification of Drosophila gene products required for phagocytosis of Candida albicans. PLoS Biol (2006) 4(1):e4. doi:10.1371/journal.pbio.0040004

78. Mathey-Prevot B, Perrimon N. Mammalian and Drosophila blood: JAK of all trades? Cell (1998) 92:697-700. doi:10.1016/S0092-8674(00)81396-3

79. Eldering M, Morlais I, van Gemert GJ, van de Vegte-Bolmer M, Graumans W, Siebelink-Stoter R, et al. Variation in susceptibility of African Plasmodium falciparum malaria parasites to TEP1 mediated killing in Anopheles gambiae mosquitoes. Sci Rep (2016) 6:20440. doi:10.1038/srep20440

80. Kamareddine L, Nakhleh J, Osta MA. Functional interaction between apolipophorins and complement regulate the mosquito immune response to systemic infections. J Innate Immun (2016) 8(3):314-26. doi:10.1159/000443883

81. Matetovici I, Caljon G, Van Den Abbeele J. Tsetse fly tolerance to T. brucei infection: transcriptome analysis of trypanosome-associated changes in the tsetse fly salivary gland. BMC Genomics (2016) 17(1):971. doi:10.1186/ s12864-016-3283-0
82. Wang YH, Hu Y, Xing LS, Jiang H, Hu SN, Raikhel AS, et al. A critical role for CLSP2 in the modulation of antifungal immune response in mosquitoes. PLoS Pathog (2015) 11(6):e1004931. doi:10.1371/journal.ppat.1004931

83. Carroll MC. Complement and humoral immunity. Vaccine (2008) 26(Suppl 8): I28-33. doi:10.1016/j.vaccine.2008.11.022

84. Xiao X, Liu Y, Zhang X, Wang J, Li Z, Pang X, et al. Complement-related proteins control the flavivirus infection of Aedes aegypti by inducing antimicrobial peptides. PLoS Pathog (2014) 10:e1004027. doi:10.1371/journal. ppat.1004027

85. Federici Canova D, Pavlov AM, Norling LV, Gobbetti T, Brunelleschi S, Le Fauder P, et al. Alpha-2-macroglobulin loaded microcapsules enhance human leukocyte functions and innate immune response. J Control Release (2015) 217:284-92. doi:10.1016/j.jconrel.2015.09.021

86. Amara U, Rittirsch D, Flierl M, Bruckner U, Klos A, Gebhard F, et al. Interaction between the coagulation and complement system. Adv Exp Med Biol (2008) 632:71-9.

87. Huber-Lang M, Sarma JV, Zetoune FS, Rittirsch D, Neff TA, McGuire SR, et al. Generation of $\mathrm{C} 5 \mathrm{a}$ in the absence of $\mathrm{C} 3$ : a new complement activation pathway. Nat Med (2006) 12:682-7. doi:10.1038/nm1419

88. Eleftherianos I, Revenis C. Role and importance of phenoloxidase in insect hemostasis. J Innate Immun (2011) 3:28-33. doi:10.1159/000321931

89. Montalto MC, Hart ML, Jordan JE, Wada K, Stahl GL. Role for complement in mediating intestinal nitric oxide synthase- 2 and superoxide dismutase expression. Am J Physiol Gastrointest Liver Physiol (2003) 285:G197-206. doi:10.1152/ajpgi.00029.2003

90. Ricklin D, Hajishengallis G, Yang K, Lambris JD. Complement: a key system for immune surveillance and homeostasis. Nat Immunol (2010) 11:785-97. doi:10.1038/ni.1923

91. Tahtouh M, Croq F, Lefebvre C, Pestel J. Is complement good, bad, or both? New functions of the complement factors associated with inflammation mechanisms in the central nervous system. Eur Cytokine Netw (2009) 20:95-100. doi:10.1684/ecn.2009.0157

92. Hollmann TJ, Mueller-Ortiz SL, Braun MC, Wetsel RA. Disruption of the $\mathrm{C} 5 \mathrm{a}$ receptor gene increases resistance to acute Gram-negative bacteremia and endotoxic shock: opposing roles of C3a and C5a. Mol Immunol (2008) 45:1907-15. doi:10.1016/j.molimm.2007.10.037

Conflict of Interest Statement: The authors declare that the research was conducted in the absence of any commercial or financial relationships that could be construed as a potential conflict of interest.

Copyright () 2017 Shokal and Eleftherianos. This is an open-access article distributed under the terms of the Creative Commons Attribution License (CC BY). The use, distribution or reproduction in other forums is permitted, provided the original author(s) or licensor are credited and that the original publication in this journal is cited, in accordance with accepted academic practice. No use, distribution or reproduction is permitted which does not comply with these terms. 